\title{
Fast wire scanner for intense electron beams
}

\author{
T. Moore, N. I. Agladze, I. V. Bazarov, A. Bartnik, J. Dobbins, B. Dunham, S. Full, \\ Y. Li, X. Liu, J. Savino, and K. Smolenski \\ Cornell Laboratory for Accelerator-Based Sciences and Education, Cornell University, \\ Ithaca, New York 14853, USA
}

(Received 10 July 2013; published 18 February 2014)

\begin{abstract}
We have developed a cost-effective, fast rotating wire scanner for use in accelerators where high beam currents would otherwise melt even carbon wires. This new design uses a simple planetary gear setup to rotate a carbon wire, fixed at one end, through the beam at speeds in excess of $20 \mathrm{~m} / \mathrm{s}$. We present results from bench tests, as well as transverse beam profile measurements taken at Cornell's high-brightness energy recovery linac photoinjector, for beam currents up to $35 \mathrm{~mA}$.
\end{abstract}

DOI: 10.1103/PhysRevSTAB.17.022801

PACS numbers: 06.60.Jn, 29.20.Ej, 29.27.Fh

\section{INTRODUCTION}

Ever increasing requirements for the quality of relativistic particle beams are the main driving force behind progress in diagnostic equipment, with new beam parameters oftentimes requiring novel approaches in beam diagnostics instrumentation. The general desire is to have a minimally interceptive (or ideally noninterceptive) online diagnostics capable of sampling the relevant beam parameters in all 6D phase space dimensions (two transverse beam positions, angles, momentum, and energy at a specific time). More specifically, size or beam profile monitors are both ubiquitous in a modern accelerator as well as necessary to push a machine's performance to its design values. Additionally, if placed in dispersive sections, beam profile monitors can measure the energy spread in the beam.

Recently, a new regime of beam parameters has become accessible with the advent of very intense photoinjectors, which feature very high average beam currents (up to $100 \mathrm{~mA}$ ) and low transverse beam emittances $(<1 \mathrm{~mm}$-mrad rms normalized) [1]. The beam energy out of the photoinjectors is near or below $10 \mathrm{MeV}$, with very large beam powers $(\sim \mathrm{MW})$ contained inside a small beam cross section (diameter of $1 \mathrm{~mm}$ or less). This parameter range poses special challenges for diagnostics. First, the beam power density is high enough that any material intercepting the beam will melt on the order of between 10 and $100 \mu$ sor less. Second, the beam energy is so low that synchrotron or diffraction radiations, which are often the method of choice for beam profile measurements at higher energies, are generally not available without introducing strong magnetic fields or placing apertures undesirably close to the beam for diffraction radiation. A

Published by the American Physical Society under the terms of the Creative Commons Attribution 3.0 License. Further distribution of this work must maintain attribution to the author(s) and the published article's title, journal citation, and DOI. diagnostics tool capable of measuring the size of these intense beams is needed with a resolution on the order of $10 \mu \mathrm{m}$. Such diagnostics would complement the interceptive low duty factor double-slit or pepper-pot measurements commonly used in photoinjectors to measure the beam's transverse phase space. Such interceptive measurements of beam emittance are required since these intense beams tend to be space-charge rather than emittance dominated. Therefore, in order to properly access the transverse phase space, the slits or the pepper-pot openings must sample the full charge per bunch beam at a much reduced repetition rate, converting the space charge dominated beam to emittance dominated beamlets that carry information on both beam position and divergence [2]. Whenever the entirety of beam physics can be explained by single bunch dynamics, such interceptive methods can be adequate. However, a number of high current phenomena can lead to large changes in the beam properties whenever the duty factor changes from a value very close to 0 , as required for the interceptive emittance measurements, to $100 \%$. These include ion accumulation and the subsequent beam charge neutralization [3] or long range wakefields either due to accelerating structures or beam pipe discontinuities. Thus, to verify the machine performance, it becomes necessary to compare measurements done both at the low duty factor and at the full power beam operation.

One obvious candidate for a beam profile diagnostic capable of measuring megawatt type electron beams is a scanning wire, which can sample the profile at a suitable transverse resolution ( $\sim \mu \mathrm{m}$ or even better) [4]. Particle beams with small transverse dimensions and high intensities impose a difficult requirement of fast scanning speed in newly designed wire scanners. State of the art instruments of this type are typically used for calibration of other less precise noninvasive transverse beam diagnostics based on synchrotron radiation imaging, beam induced gas luminescence observation, and conventional beam position monitors [5-7]. 
The Cornell photoinjector, built as a prototype electron source of a large x-ray facility [8], has recently operated with beam powers approaching 0.5 MW (maximum design current of $100 \mathrm{~mA}$ at $5 \mathrm{MeV}$ beam energy) [1,9]. The bunch frequency is $1.3 \mathrm{GHz}$ with the bunch charge corresponding to $77 \mathrm{pC}$ for $100 \mathrm{~mA}$ average current. A wire scanner operating at a sufficiently high speed is one of the minimally invasive transverse beam diagnostics suitable for such intense beams. Carbon wires made from a precursor polymer material and graphitized in vacuum at the temperature $2300 \mathrm{~K}$ are one of the best materials for withstanding extremely high temperature rises (up to $3600 \mathrm{~K}$ ) during a scan [10]. As found from experiment, these carbon fibers break after depositing $10^{10}$ electrons onto a $3 \mu \mathrm{m}^{2}$ area [11]. For a beam current of $100 \mathrm{~mA}$ and both an electron beam diameter and a wire diameter of $34 \mu \mathrm{m}$, this results in the minimal scanning speed of $20 \mathrm{~m} / \mathrm{s}$. This limitation comes primarily from the beam energy and the transverse dimension since according to the first-order model the wire temperature rise does not depend on its diameter [6]. This is the same speed needed for a safe operation of wire scanners located at the interaction point of the LHC [10].

In this paper, we describe a simplified wire scanner design optimized for a large quantity reproduction resulting in a significant reduction of the cost. The main idea of the design is to use a carbon fiber fixed at one end, enabling a scanning speed in excess of $20 \mathrm{~m} / \mathrm{s}$ with a simple and low moment of inertia planetary gear setup made from readily available standard components. Results of bench tests and applications of the new wire scanner prototype in the Cornell energy recovery linac (ERL) photoinjector-operating at beam energy $4 \mathrm{MeV}$, bunch frequency $1.3 \mathrm{GHz}$, and currents up to $35 \mathrm{~mA}$ - are presented in this paper.

\section{WIRE SCANNER CONSTRUCTION}

The principle of operation of the new wire scanner is illustrated in Fig. 1. It consists of a stationary gear $G_{1}$ and a smaller gear $G_{2}$ rotating around the gear $G_{1}$. The blade attached to the gear $G_{2}$ holds the carbon fiber cemented on its end. If the center of the small gear moves with the linear velocity $v_{g}$ then the fiber scanning speed is

$$
v_{S}=v_{g}\left(\frac{R}{R_{2}}+1\right)
$$

where $R_{2}$ is the radius of the gear $G_{2}$ and $R$ is the distance from the center of the gear $G_{2}$ to the electron beam. Uncertainty of the beam position along the fiber results in the scanning speed variation

$$
\frac{\delta v_{s}}{v_{s}}=\frac{\left(\delta R / R_{2}\right)+1}{\left(R / R_{2}\right)+1} \approx \frac{\delta R}{R},
$$

where it is assumed that $\delta R \ll R, R_{2}$.In the current prototype $R=82.4 \mathrm{~mm}$ so for $\delta R=5 \mathrm{~mm}$ the relative scanning

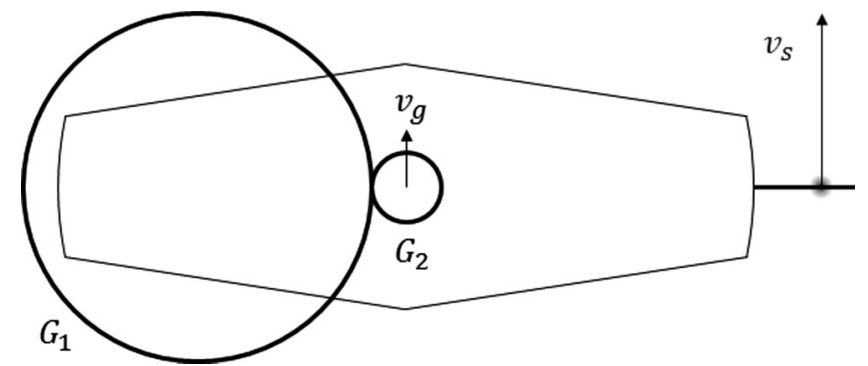

FIG. 1. A schematic representation of the wire scanner operation. The gear $G_{2}$ with the blade attached to it rotates around the stationary gear $G_{1}$. The carbon fiber at the end of the blade intercepts the beam, producing a time-dependent signal proportional to the beam projection on a direction orthogonal to the fiber.

speed variation is $\delta v_{s} / v_{s}=0.061$.This value sets the absolute precision of the transverse bunch profile determination.

The photograph of the wire scanner is shown in Fig. 2 and the gears setup is shown in Fig. 3. The only custommade parts are the aluminum gear box and the fiber holding blade. These are installed between a pair of standard high vacuum flanges with $337 \mathrm{~mm}$ outer diameter and an inner hole with $152 \mathrm{~mm}$ diameter. Another set of two vacuum flanges of diameter $203.2 \mathrm{~mm}$ enclose the setup. One of the flanges is equipped with three optical view ports for monitoring the carbon fiber's condition between scans. The rotation is provided by a ferrofluidic rotary feedthrough (Thermionics FRMRE-275-38CL) driven by a stepper motor. The maximum scanning speed is determined by the moment of inertia of the rotating parts (motor rotor, rotary feedthrough, gear box, and the blade), the friction in the gears and ball bearings, and the maximum torque rating of the rotary feedthrough. The gears and ball bearings were lubricated with Dicronite-a high vacuum compatible

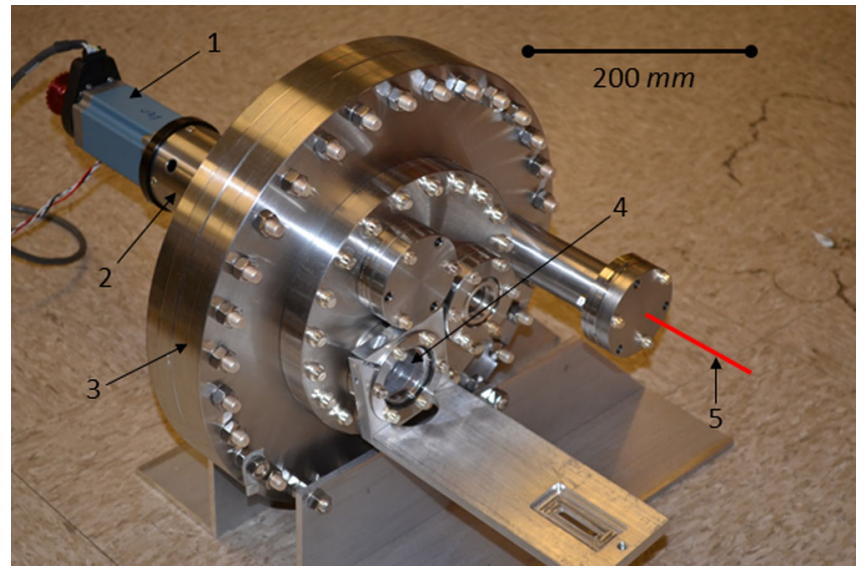

FIG. 2 (color online). A photograph of the wire scanner: (1) stepper motor; (2) ferrofluidic rotary feadthrough; (3) vacuum flanges; (4) vacuum flange with viewports; (5) beam direction. 


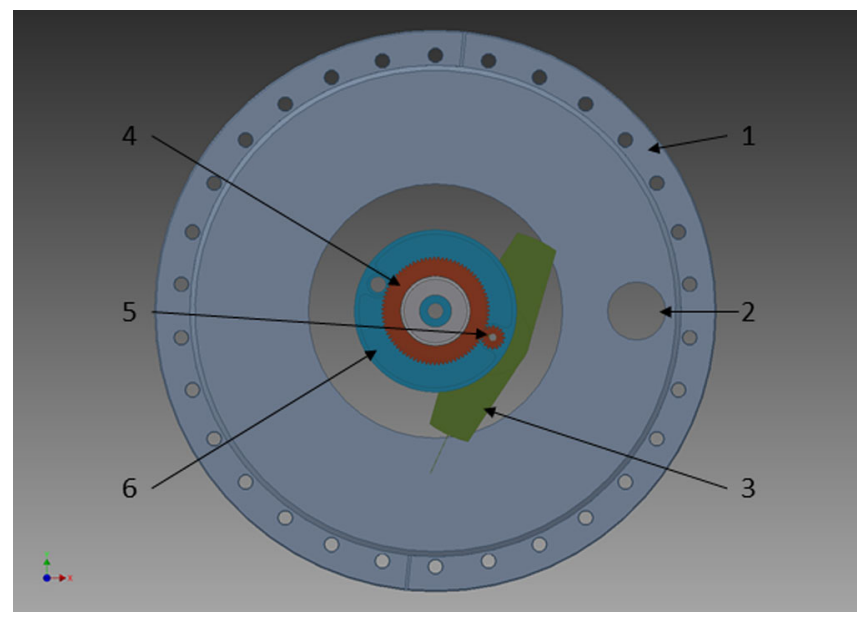

FIG. 3 (color online). A 3D rendering of the gears setup: (1) vacuum flange; (2) beam pipe; (3) blade with attached carbon wire; (4) stationary gear $G_{1}$; (5) rotating gear $G_{2}$; (6) rotating gear box.

lubricant. Bench tests confirmed that mechanical friction is sufficiently small, and, with the existing moment of inertia of the system and torque limit of the feedthrough $(1.06 \mathrm{Nm})$ scanning speeds can reach up to $30 \mathrm{~m} / \mathrm{s}$. For our experiments we only operated at $20 \mathrm{~m} / \mathrm{s}$ as this speed adequately fulfilled our design requirements. In addition, the magnetic rotary feedthrough we used began to slip for speeds above $20 \mathrm{~m} / \mathrm{s}$, forcing us to recalibrate our device between measurements and giving us further reason to operate at slower speeds.

The starting position at the beginning of the scan is chosen so that the fiber is located slightly above the edge of the beam pipe, while taking into account the slight bending due to gravity. During the acceleration stage, the gear $G_{2}$ makes an almost complete revolution around the gear $G_{1}$ and the carbon fiber reaches its maximum velocity when it crosses the center of the beam pipe. Afterwards the mechanism is decelerated and the fiber is stopped just below the beam pipe, opposite its starting position. Before the next measurement is taken, the wire is returned to its initial starting position by moving it at a slow, constant velocity in the opposite direction, so that it does not pass through the beam pipe. The abrupt deceleration of the wire when it arrives at its starting position causes the wire to vibrate- a problem we address later in this paper. In order to avoid abrupt changes in the wire's acceleration during a scan, which lead to excessive vibrations, the velocity profile is programmed to follow a smooth sinusoidal curve for a nominal scanning speed of $20 \mathrm{~m} / \mathrm{s}$. The full motion takes less than $700 \mathrm{~ms}$, with the maximum speed attained in the center of the beam pipe. It takes $2 \mathrm{~ms}$ to scan across the beam pipe and the speed at the edges drops only by $0.0005 \mathrm{~m} / \mathrm{s}$, even without creating the constant speed segment in the velocity profile.

\section{BENCH TESTS}

In contrast to other wire scanners described in the literature [4-7], the carbon fiber is attached (cemented using electrically conducting epoxy) to the rotating blade only at one end, so that a large amplitude vibration-up to $1 \mathrm{~mm}$ or more - can be easily excited. This should be compared to typical wire vibration amplitudes not exceeding $10 \mu \mathrm{m}$ in instruments with stretched wires fixed at both ends [12]. A previous design using a wire attached to one end was employed successfully in 1969 at Brookhaven's Alternating Gradient Synchrotron (AGS) [13]. While they utilize a somewhat similar planetary gear design, their scheme relied on flipping the wire through the beam using a free rotating cylinder. This prevents them from programing their wire's velocity profile, thereby resulting in significantly less control of the wire during a scan. Nevertheless, they claim that vibrations were not an issue for wire speeds up to $6.1 \mathrm{~m} / \mathrm{s}$. However, this lack of control creates a need for our updated design to further minimize vibrations, control the wire before and after a scan, and most importantly reach higher scanning speeds. Finally, it should also be noted that the knowledge of the average position of the beam, which can be compromised by such vibrations, is of a lesser concern when noninterceptive beam position monitors are readily available in accelerators with resolutions of $10 \mu \mathrm{m}$ and better.

In the harmonic approximation the amplitude of the wire oscillation in the scanning direction is $A(x)=A_{0}(x)$ $\sin (\omega t+\varphi)$, where the oscillation is assumed to be of the lowest order with all points of the wire having the same phase. The corresponding speed is then $v(x)=\omega A_{0}(x) \cos$ $(\omega t+\varphi)$. This oscillation speed will add to the wire scanning speed and as a result can affect the beam profile measurement. In the prototype instrument the oscillation frequency of the wire $\omega / 2 \pi=75 \pm 2 \mathrm{~Hz}$ was determined experimentally by shining a laser onto the vibrating wire and tuning the laser pulse frequency until the image of the wire, obtained using a standard charge-coupled device (CCD) camera, became stationary. Given the maximum amplitude of the oscillation $A_{0}=5 \mathrm{~mm}$ (as evidenced by direct observation with the video camera), the wire scanning speed can be modulated by up to $\omega A_{0}=2.4 \mathrm{~m} / \mathrm{s}$.

In order to verify these estimates, the bench test schematically presented in Fig. 4 was performed. The idea is to use a modulated laser to observe multiple images of the moving wire in a single CCD frame. This is achieved by suppressing the background illumination with the aid of two Fourier transform lenses and a spatial filter. Laser light not scattered by the wire (1) is focused by the lens (2) and blocked by the obstruction (3) (a stripe of black electrical tape parallel to the wire). The laser light scattered by the wire is not blocked by the obstruction (3) since it is collimated by the lens (2). The lens (4) then collects the scattered light and creates the wire image (5) at the surface of the CCD sensor. The wire scanner was evacuated to a 


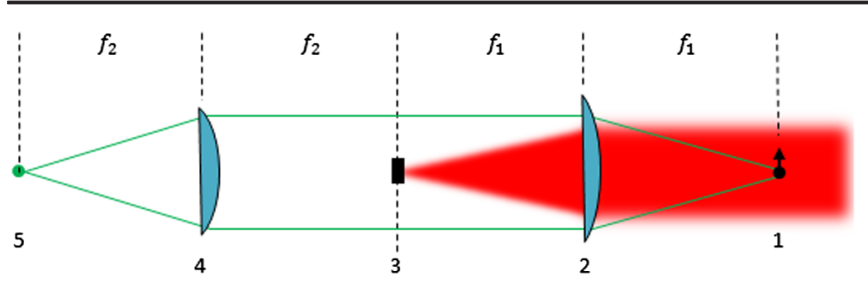

FIG. 4 (color online). Optical schematics for a bench test designed to take images of the wire in motion. (1) moving wire illuminated by a pulsed diode laser; (2) first Fourier transform lens, (3) spatial filter that removes the zero order laser light; (4) second Fourier transform lens; (5) image of the moving wire formed by the scattered laser light, observed with a CCD camera (not shown).

pressure of approximately $10^{-7}$ Torr during these tests, and the laser beam was sent through the beam pipe using two flanges with optical windows. We expected that during rotation the gears and magnetic rotary feedthrough would cause a spike in vacuum pressure, and indeed this was the case. However, the pressure spike was $10^{-7}$ Torr at most, which is sufficiently low for successful beam operation in our case.

An example of the wire imaging is shown in Fig. 5. The tilt of the image is due to the wire bending under gravity. By modulating the laser, we are able to capture several images of a moving wire on a single camera frame, for a sufficiently fast modulation frequency and large enough exposure time. During the exposure time wire oscillations manifest as a slight broadening of the image. Figure 6 shows the frame obtained for a wire moving at $20 \mathrm{~m} / \mathrm{s}$ using a laser pulse duration $7 \mu \mathrm{s}$ and a pulse frequency $8 \mathrm{kHz}$. The laser light scattered by optical imperfections is removed by subtracting the frame without the wire. The tilt that was observed in the wire at rest is slightly reduced during motion due to the centripetal force action. Vertical

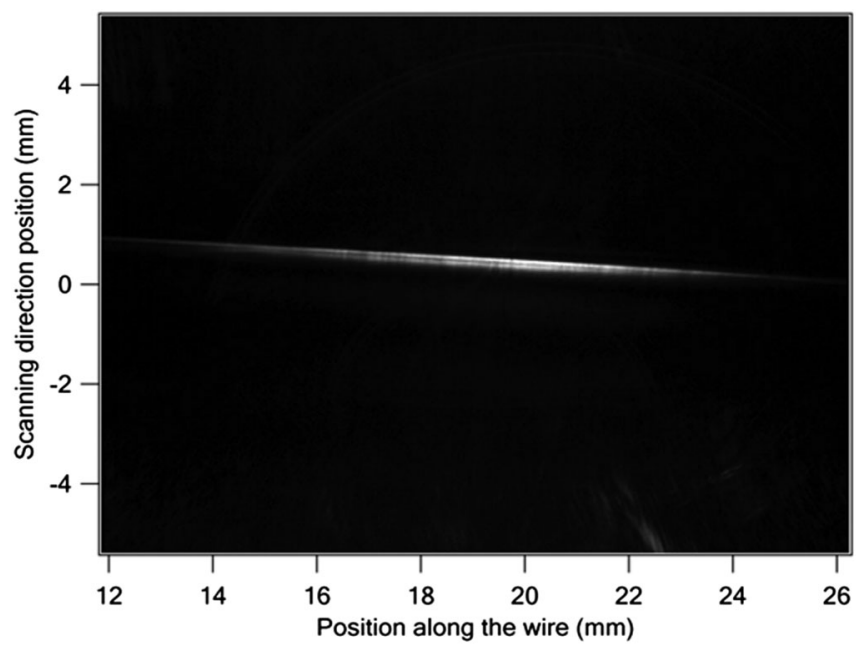

FIG. 5. Image of the carbon wire at rest taken during the bench test depicted in Fig. 4.

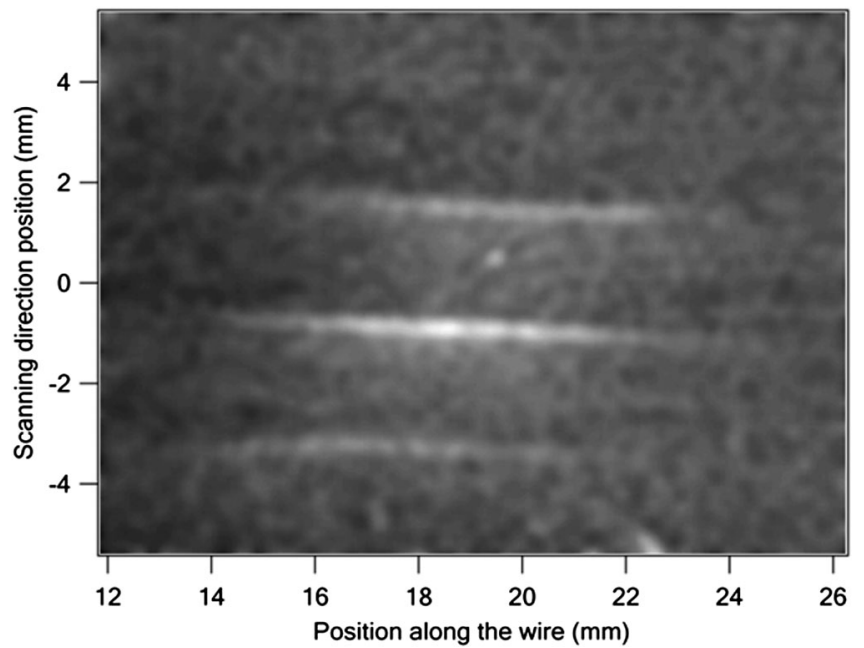

FIG. 6. Three images of the wire moving at a scanning speed of $20 \mathrm{~m} / \mathrm{s}$. Movement is in the upward direction, and the laser pulse frequency is $8 \mathrm{kHz}$. The wires are attached to the rotating blade on the left.

image profiles shown in Fig. 7 illustrate the difference in the movement of various wire points. Each profile corresponds to the certain distance from the wire holding blade, and the profiles are obtained by averaging 50 pixels $(0.37 \mathrm{~mm})$ along the wire. The center of the beam pipe is chosen as the origin and the edges of the pipe are located at $\pm 19 \mathrm{~mm}$. The precise positions of peaks (1)-(3) were determined by fitting Gaussian profiles near the maxima. The wire speed was calculated from the distance between adjacent wire images in the scanning direction, and by using our knowledge of the laser modulation frequency and

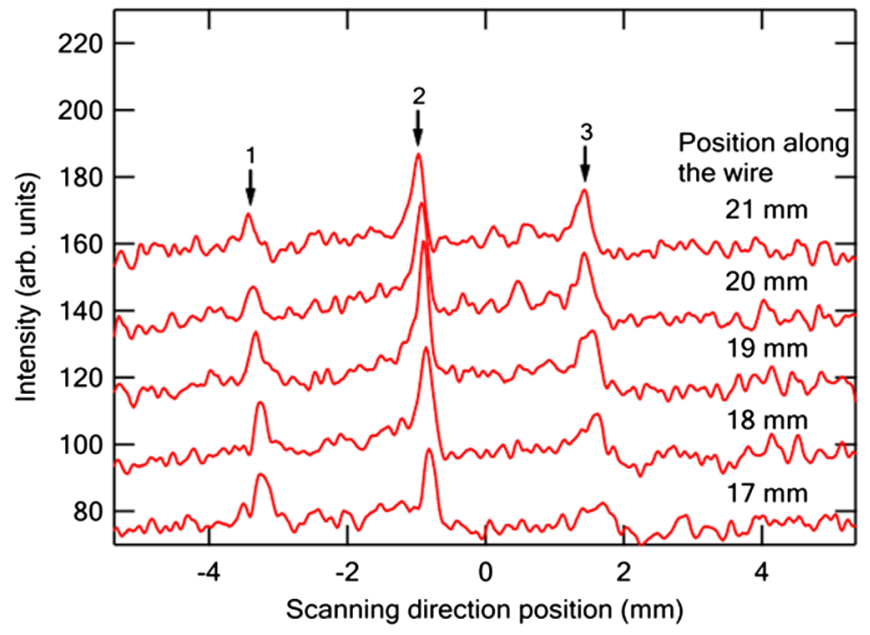

FIG. 7 (color online). Vertical profiles at different locations along the wire obtained by taking vertical slices at several horizontal positions of the image in Fig. 6 (horizontal axis corresponds to the vertical axis in Fig. 6). Peaks (1), (2), and (3) are used for determining the distance between adjacent wire images and for subsequently calculating the wire's speed. 


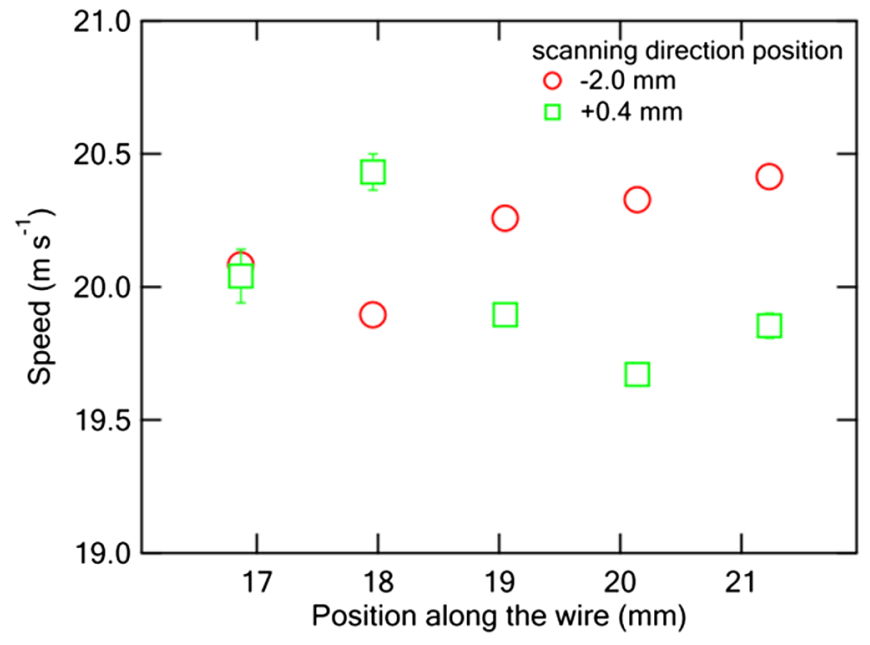

FIG. 8 (color online). Wire speeds calculated at different locations along the wire and at two different scanning positions. The circles represent values calculated using the average between peaks (1) and (2), while the squares use peaks (2) and (3). The error bars are smaller than the symbol size for some of the points.

magnification of our optical system. The laser modulation frequency is fixed and well known, but the resolution of our measurement is limited by the magnification of the system, which in turn is limited by the need to capture at least three images of a wire on a single camera frame. Results are shown in Fig. 8. Wire speed variations are observed both along the wire and in the scanning direction, pointing to the presence of the higher order vibrations. The average speeds determined at two wire scanning positions $-2.0 \mathrm{~mm}$ and $0.4 \mathrm{~mm}$ are $20.2 \pm 0.2 \mathrm{~m} / \mathrm{s}$ and $20.0 \pm 0.3 \mathrm{~m} / \mathrm{s}$, respectively. The uncertainty is well within the estimate

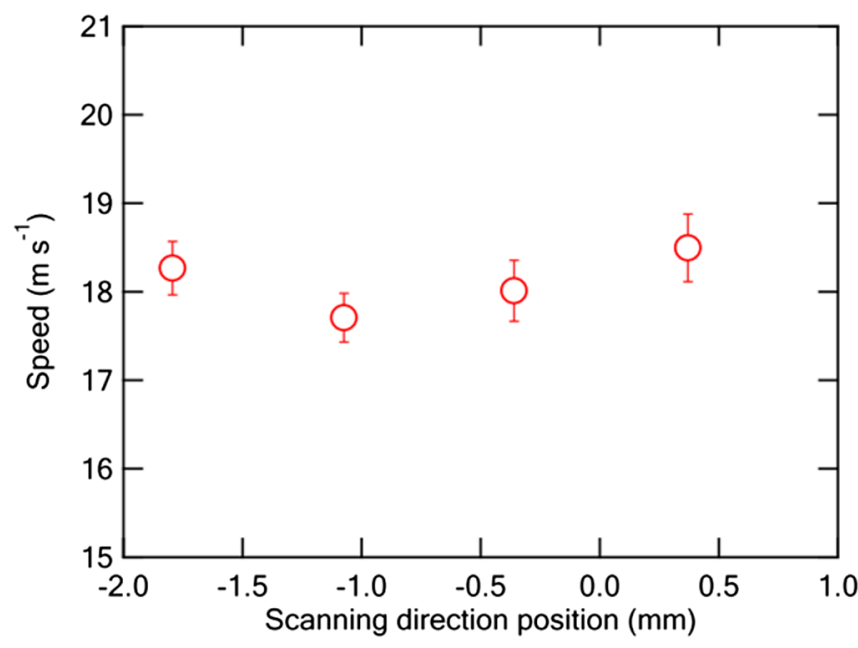

FIG. 9 (color online). Wire speed variation along the scanning direction determined from 5 profiles obtained with $25 \mathrm{kHz}$ laser modulation. The scanning speed was calculated by measuring the distance between adjacent wire images, at the location where the wire intersects the center of the beam pipe. of $2.4 \mathrm{~m} / \mathrm{s}$, based on the assumption that the amplitude of the fundamental wire vibration is less than $5 \mathrm{~mm}$.

A more detailed measurement of wire speed variations during the scan was obtained by modulating the laser at $25 \mathrm{kHz}$ for a wire scanning speed $18 \mathrm{~m} / \mathrm{s}$. In this test the diameter of the expanded laser beam enabled observation of up to five well-resolved images of the moving wire. The scanning speed was then calculated by measuring the distance between adjacent wire images, at the location where the wire intersects the axis of the beam pipe. Results are shown in Fig. 9, and the average value of the speed was found to be $18.1 \pm 0.3 \mathrm{~m} / \mathrm{s}$. This is consistent with the data obtained at $8 \mathrm{kHz}$ laser modulation, and the conclusion is that the wire scanning speed uncertainty is $\frac{0.3 \mathrm{~m} / \mathrm{s}}{20 \mathrm{~m} / \mathrm{s}}=0.015$, or $1.5 \%$.

\section{BEAM TESTS}

Beam tests of the new wire scanner were performed after installation in the chicane section of the Cornell ERL photoinjector [9]. $\mathrm{X}$ rays produced when the wire crosses the electron beam were detected using a combination of a scintillator crystal and a silicon photomultiplier sensor (MicroSM-60035-X13 by SensL). A standard power supply and preamplifier (Micro-EVB) from the same manufacturer were used for signal conditioning and the bias supply necessary for operation of the silicon photomultiplier. The number of x-ray photons produced during the wire scan was sufficiently high to saturate the detector. By reducing the number of optical photons produced by each $\mathrm{x}$-ray photon, the dynamic range of the detector can be extended without sacrificing a low-noise level. For this purpose, a neutral density filter (attenuation $\approx 30$ ) was introduced between the scintillator and the detector. It enabled reliable operation of the detection system without saturation while maintaining a sufficiently high signal-tonoise ratio for all experimentally tested beam currents (1-35 mA).

The detector output was sampled at a rate of $2 \times 10^{6}$ samples per second with the aid of a digital acquisition device (Agilent U2500A USB DAQ). At a scanning speed of $20 \mathrm{~m} / \mathrm{s}$, this corresponds to a distance sampling interval of $10 \mu \mathrm{m}$, compared to the $34 \pm 1 \mu \mathrm{m}$ diameter of the carbon fiber used in these experiments. Data acquisition can be triggered either by the stepper motor controller (DMC2183 by Galil Motion Control) at a preset wire position, or by the control software after a fixed time interval.

The rf field excited in the wire scanner housing by the electron beam and subsequently absorbed by the conducting wire can potentially lead to its destruction even without scanning [14]. The solution to this problem was to use a blade made from a silica wafer with a metal absorbing layer. By matching the square resistance of the layer to the vacuum impedance, the broadband absorption of the $\mathrm{rf}$ field was achieved, preventing the appearance of standing waves in the housing. Nevertheless, in the experiments described 
here this silica blade was not used since no rf absorption effects were detected with a simpler aluminum blade up to $35 \mathrm{~mA}$ beam current. In addition, we have calculated a loss factor of $3.0 \pm 0.5 \mathrm{~V} / \mathrm{pC}$ by approximating the wire scanner as a pillbox cavity [15]. For a $77 \mathrm{pC}$ beam operating at $100 \mathrm{~mA}$, this corresponds to an energy loss of approximately $23 \mathrm{~W}$, which is negligible for our beam, but potentially could result in the wire destruction. Higher beam current performance will be evaluated soon. Radio frequency absorbers can be easily incorporated in our present design should it be necessary.

Beam tests at an energy of $4 \mathrm{MeV}$, a fixed beam current of $5 \mathrm{~mA}$, and a bunch frequency of $1.3 \mathrm{GHz}$ were performed to investigate the effects of the wire vibrations, excited when abruptly stopping the wire between scans, on beam profile measurements. Specifically, the wire vibrated every time it was moved to the starting position for a new scan, and the amplitude of these vibrations decayed slowly over time. The damping time constant $\tau=60 \pm 5 \mathrm{~s}$ was experimentally measured by observing the amplitude of the oscillation with a CCD camera. The first set of runs was performed with approximately a $3 \tau$ pause before starting the next scan to allow time for the wire vibration amplitude to decrease to a negligible value. For each measured beam profile, the full width at half maximum (FWHM) was determined from a smoothing spline fit. Results are shown in Fig. 10.

The second set of measurements was performed with only $20 \mathrm{~s}$ between moving the wire to starting position and beginning the scan. Some residual vibration was expected in this case. These results are shown in Fig. 10. A small difference in beam profile reproducibility is observed between these two series of measurements. For both series, the calculated $\mathrm{FWHM}=2.79 \mathrm{~mm} \pm 0.13$, corresponding to a relative precision $4.7 \%$, which is larger than the wire speed variations due to the vibrations found in the bench tests. This can either be explained as an actual change in beam size during our tests, or a direct result of residual wire vibrations. As this is presently our only beam profile diagnostic for high current operations, we are unable to determine the former. If it is truly an issue, the latter can be corrected for by more smoothly returning the wire to its starting position to avoid abrupt accelerations. However, our bench test results suggest that vibrations are not the primary issue.

In order to test the dynamic range of the instrument, several sets of tests were performed for beam currents varying from $250 \mu \mathrm{A}$ to $35 \mathrm{~mA}$. The measured beam profiles were normalized by subtracting an offset and dividing the amplitude by the value of the current in $\mathrm{mA}$. Results are shown in Fig. 11. The total area under the curve is preserved, confirming the good linearity of the detection system. As the bunch charge is increased, a systematic broadening of the profile is observed, which can be explained as a result of space charge repulsion.

The possible distortion of the profile due to transient effects in the detection system was tested by running the

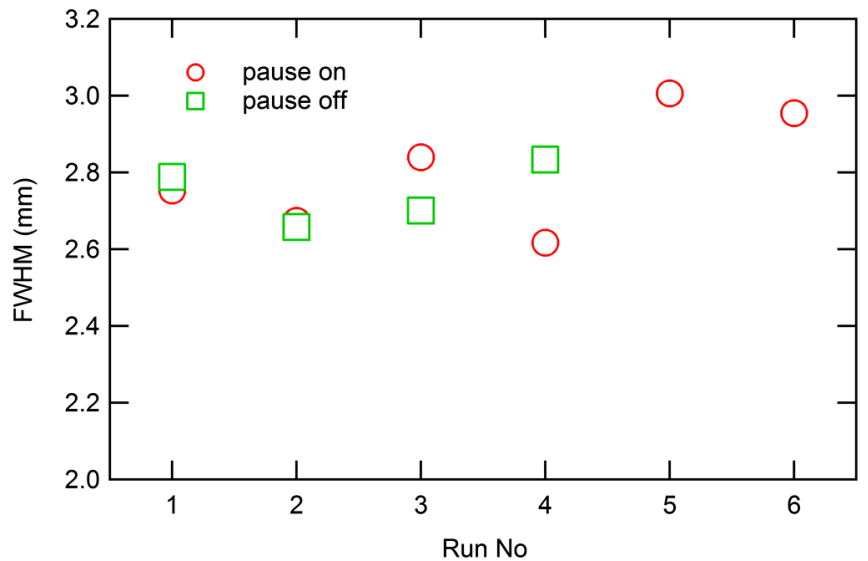

FIG. 10 (color online). Variations of the beam profile FWHM at beam current $5 \mathrm{~mA}$ measured with the prototype wire scanner. Circles denote set of runs with a $180 \mathrm{sec}$ pause before the scan onset for the wire to stop vibrating; squares denote set of runs with a $20 \mathrm{sec}$ pause (pause off) before the scan with the wire still vibrating. Error bars are smaller than the symbol size.

wire in the opposite direction. Figure 12 shows a comparison of two profiles acquired at a $35 \mathrm{~mA}$ beam current with the wire moving in the upward direction (solid line) and in the downward direction (broken line). Both profiles are reproduced with a sufficient precision confirming that the observed beam asymmetry is real and not due to detection system artifacts.

At low beam currents the profiles can be obtained using a luminescent viewscreen. Figure 13 shows a comparison of the viewscreen and wire scanner profiles measured for the same beam current of $20 \mu \mathrm{A}$, obtained by decreasing

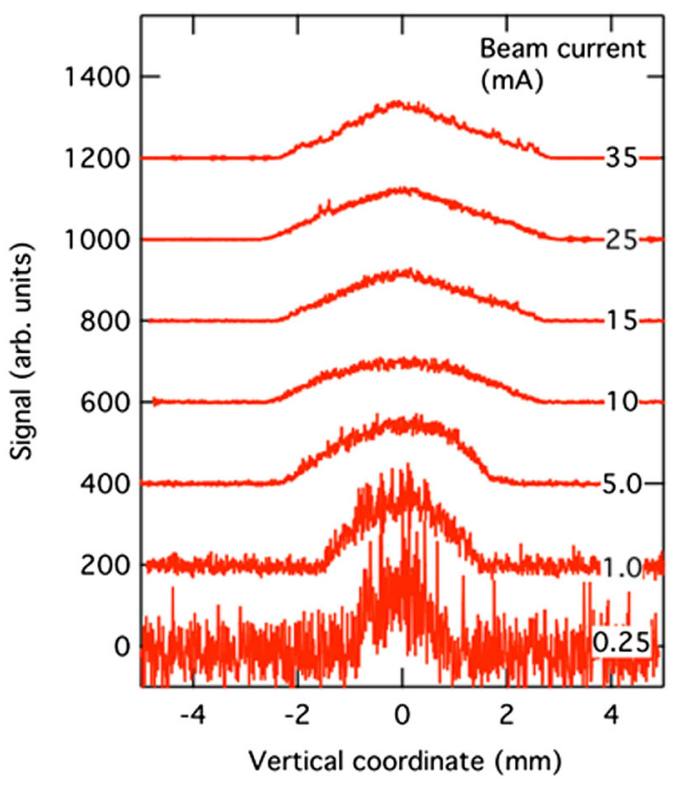

FIG. 11 (color online). Wire scans at different beam currents. Data are normalized to $1 \mathrm{~mA}$ current. 


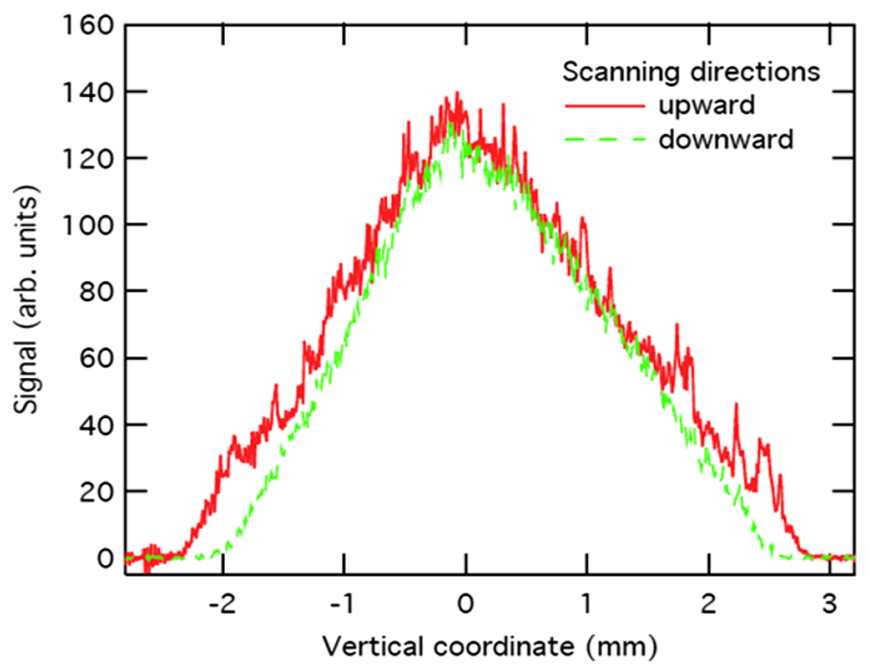

FIG. 12 (color online). Wire scans in opposite directions. Solid line denotes wire moving in the upward direction; broken line denotes wire moving in the downward direction.

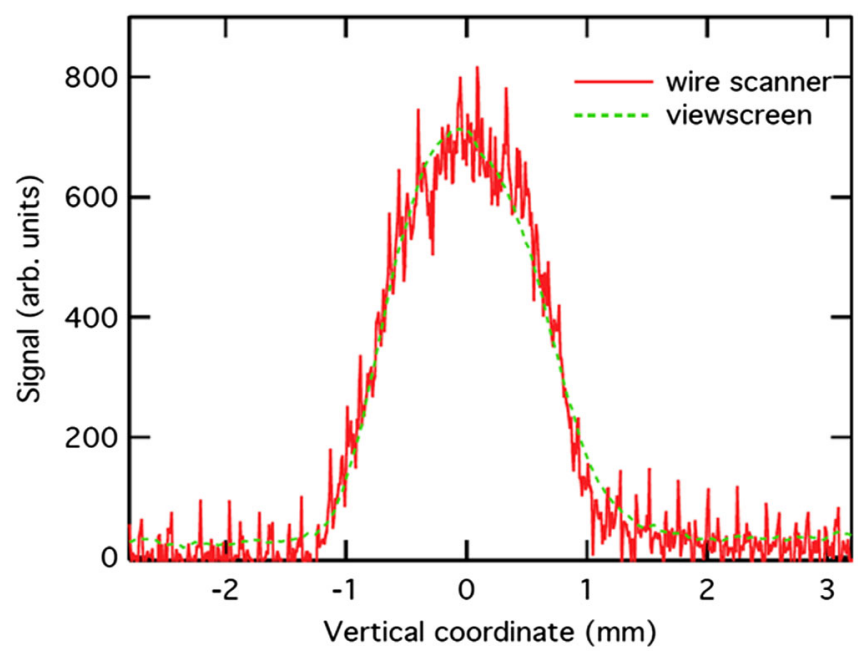

FIG. 13 (color online). Comparison of the beam profile obtained with the wire scanner (solid line) and the luminescent viewscreen (broken line) for $20 \mu \mathrm{A}$ average beam current (0.015 pc bunch charge).

bunch charge to $0.015 \mathrm{pC}$. A regular beam loss monitor photomultiplier was used for the wire scanner signal detection this time. Both curves are in very close agreement, confirming that both diagnostics are consistent for low bunch charge values.

\section{CONCLUSIONS}

In conclusion, both bench tests and high current beam experiments were performed using the simplified fast-rotating wire scanner operating at a speed of $20 \mathrm{~m} / \mathrm{s}$. It is capable of measuring beam profiles in one plane with a resolution as low as $10 \mu \mathrm{m}$ and a relative precision of $1.5 \%$ of the measured FWHM beam size, and can be used in a wide range of currents from $250 \mu \mathrm{A}$ to $100 \mathrm{~mA}$ (up to $35 \mathrm{~mA}$ experimentally verified). This diagnostics can be used to characterize a number of beam effects arising in a new class of high intensity low energy electron accelerators, which are not easily accessible with alternative methods.

\section{ACKNOWLEDGMENTS}

This work was supported by the financial assistance from the National Science Foundation (Grant No. DMR0807731).

[1] C. Gulliford et al., arXiv:1304.2708v1.

[2] I. V. Bazarov, B. M. Dunham, C. Gulliford, Y. L. Li, X. H. Liu, C. K. Sinclair, K. Soong, and F. Hannon, Phys. Rev. ST Accel. Beams 11, 100703 (2008).

[3] M. Reiser, Theory and Design of Charged Particle Beams (Wiley-VCH, Weinheim, 2008), 2nd ed.

[4] P. Tenenbaum and T. Shintake, Annu. Rev. Nucl. Part. Sci. 49, 125 (1999).

[5] B. Dehning, J. Emery, J. Herranz Alvarez, M. Koujili, and J. L. Sirvent Blasco, Proceedings of the Beam Instrumentation Workshop 2012, Newport News, VA (Jefferson Laboratory, Newport News, VA, 2012), pp. 021-023, TUPG029 [http://epaper.kek.jp/BIW2012/papers/tupg029.pdf].

[6] K. Wittenburg, Report No. TESLA2000-18, 2000 [http:// tesla.desy.de/new_pages/TESLA_Reports/2000/pdf_files/ tesla2000-18.pdf].

[7] J. Wu, P. Emma, and R. C. Field, Report No. SLAC-PUB12120, 2006 [http://www.slac.stanford.edu/pubs/slacpubs/ 12000/slac-pub-12120.html].

[8] D. H. Bilderback, J. D. Brock, D. S. Dale, K. D. Finkelstein, M. A. Pfeifer, and S. M. Gruner, New J. Phys. 12, 035011 (2010).

[9] B. Dunham et al., Appl. Phys. Lett. 102, 034105 (2013).

[10] M. Sapinski, Report No. CERN-AB-2008-030-BI, 2008 [http://cds.cern.ch/record/1123363/files/].

[11] C. Field, Nucl. Instrum. Methods Phys. Res., Sect. A 360, 467 (1995).

[12] N. Iida, T. Suwada, Y. Funakoshi, T. Kawamoto, and M. Kikuchi, Proceedings of the APAC98, KEK, Japan (KEK, Tsukuba, Japan, 1998) [http://accelconf.web.cern.ch/ AccelConf/a98/APAC98/5D061.PDF].

[13] U. Vogel, IEEE Trans. Nucl. Sci. NS-16, 905 (1969).

[14] F. Caspers, B. Dehning, E. Jensen, J. Koopman, J. F. Malo, and F. Roncarolo, Proceedings of the DIPAC 2003, Mainz, Germany (GSI, Darmshtadt, 2003), pp. 119-121 [http:// accelconf.web.cern.ch/Accelconf/d03/papers/PM12.pdf].

[15] A. Blednykh and S. Krinsky, Phys. Rev. ST Accel. Beams 13, 064401 (2010). 